\title{
Deterioro en la actividad ventilatoria hipóxica inducido por neuropatía autonómica diabética, una causa de diagnóstico erróneo de eventos cardiacos graves: breve reporte de dos casos
}

\author{
Louis Schubert $^{a}$ Suzanne Laroche ${ }^{a}$ Agnès Hartemann ${ }^{a, b}, c, d$ Olivier Bourron ${ }^{a}, b, c, d$ \\ Franck Phan ${ }^{a, b}, c, d$ \\ a Departamento de Diabetología, Hospital Pitié-Salpêtrière-Charles Foix, AP-HP, París, Francia; \\ bSorbonne Université, París, Francia; CINSERM, UMR_S 1138, Centre de Recherche Des Cordeliers, París 06, Francia; \\ dInstituto de Cardiometabolismo y Nutrición ICAN, París, Francia
}

\author{
Palabras clave \\ Insuficiencia respiratoria · Resucitación cardiopulmonar . \\ Neuropatía autonómica cardiaca · Hipoxia · Diabetes mellitus tipo 1
}

\section{Resumen \\ Resumen: La ocurrencia de muerte súbita cardiaca es dos veces más frecuente en pacientes diabéticos con neuropatía autonómi- ca. La etiología de la muerte súbita cardiaca aún no está clara, y no existen recomendaciones para identificar factores asociados con el paro cardiorrespiratorio en pacientes diabéticos. Proponemos la hipótesis, con base en dos casos clínicos, de que un deterioro en la actividad respiratoria hipóxica, inducido por la neuropatía autonó- mica diabética, es una causa del diagnóstico erróneo de eventos cardiacos graves.}

Presentación de casos: Describimos los casos de dos pacientes con baja saturación sanguínea aislada, medida con oxímetro de pulso durante la revisión rutinaria (77\% y $85 \%$, respectivamente), que contrasta con la ausencia de molestias como disnea, polipnea u otros signos de insuficiencia respiratoria durante el examen clínico. La gasometría arterial confirmó subsecuentemente la baja saturación de oxígeno en la sangre, y que ambas pacientes se hallaban hipoxémicas. La paciente 1 sufría una sobrecarga vascular complicada con paro cardiaco causado por hipoxemia, en vista de la rápida recuperación observada después de la ventilación. En la paciente 2 se diagnosticó edema pulmonar. El denominador común en los dos casos descritos en este breve reporte es la ausencia de signos clínicos de insuficiencia respiratoria y su contraste con la

information@karger.com www.karger.com/kxn

Karger ${ }^{\prime \prime}=$ 马OPEN ACCESS (c) 2021 The Author(s)

Published by S. Karger GmbH, Freiburg

This article is licensed under the Creative Commons Attribution 4.0 International License (CC BY) (http://www.karger.com/Services/OpenAccessLicense). Usage, derivative works and distribution are permitted provided that proper credit is given to the author and the original publisher. presencia confirmada de hipoxemia. Asimismo, en ambos casos, la ausencia de dichos signos precursores parece deberse a un deterioro en la actividad ventilatoria hipóxica, que resultó en hipoxemia. Ésta parece estar relacionada con la neuropatía autonómica diabética encontrada en estas dos pacientes.

Conclusiones: Por tanto, en este breve reporte señalamos a la neuropatía autonómica cardiaca como una causa de deterioro en la actividad respiratoria hipóxica, vinculado con eventos cardiorrespiratorios agudos y severos en dos pacientes con diabetes tipo 1. Asumimos que una respuesta alterada a la hipoxemia debida a la neuropatía autonómica cardiaca y un comando respiratorio neurológico central no-funcional podría tener un papel clave en la muerte súbita entre pacientes diabéticos. Un punto importante es que es fácil pasar por alto la hipoxemia, puesto que no se reportan signos de insuficiencia respiratoria en estos dos casos clínicos. Es necesario aplicar una búsqueda sistemática de neuropatía autonómica cardiaca y detectar de manera proactiva el deterioro en la actividad respiratoria hipóxica en pacientes diabéticos, para garantizar un manejo temprano de la condición (p. ej., dando tratamiento a la hipoxia) y prevenir consecuencias dramáticas, como el paro cardiorrespiratorio y la muerte.

(c) 2021 Schubert, Laroche, Hartemann, Bourron, Phan
Correspondencia:

Olivier Bourron, olivier.bourron@ aphp.fr 


\section{Antecedentes}

Entre las complicaciones de la diabetes, se ha considerado que la neuropatía es no-letal, y con frecuencia no se le evalúa con cuidado. Varios estudios revelan que sólo alrededor de $65 \%$ de los pacientes diabéticos se someten a una evaluación de neuropatía mediante la prueba con monofilamento de $10 \mathrm{~g}$ [1], y ciertamente muchos menos se sujetan a una evaluación de neuropatía autonómica cardiaca (NAC). Los índices de prevalencia reportados para la NAC varían grandemente de acuerdo con los criterios empleados para definirla, así como con las características de la población investigada. La prevalencia de NAC varía desde valores tan bajos como $2.5 \%$ en la cohorte de prevención primaria en el Ensayo de Control y Complicaciones de la Diabetes (DCCT) [2] hasta otros tan altos como $90 \%$ en pacientes con diabetes tipo 1 de larga duración que eran candidatos potenciales para trasplante de páncreas [3]. El tamizado para detectar NAC entre pacientes con diabetes representa un problema importante para la predicción de la recurrencia del síncope. De hecho, la NAC diabética está asociada con una tasa mayor de recurrencia de síncope vaso-vagal en un estudio de seguimiento por 12 meses [4]. Más aún, otros estudios sugieren que la tasa de mortalidad en pacientes diabéticos es mayor entre aquellos con neuropatía diabética establecida, particularmente NAC, que en quienes no la padecen [5]. Cabe señalar que, si bien la muerte súbita cardiaca (MSC) es la principal causa del aumento en la tasa de mortalidad de pacientes diabéticos que padecen NAC, los mecanismos de la MSC podrían ser múltiples y aún no se conocen con claridad.

Las manifestaciones clínicas de la NAC son numerosas. Algunas son menores, como los síndromes de taquicardia en reposo, intolerancia al ejercicio, taquicardia ortostática y bradicardia. Algunos otros pueden llevar a complicaciones, como inestabilidad cardiovascular intra- y perioperatoria, hipotensión ortostática, isquemia del miocardio silenciosa o cardiopatía autonómica asociada con disfunción diastólica del ventrículo izquierdo [5]. Ewing et al. [6] propusieron la aplicación de cinco pruebas en 1985 para diagnosticar NAC en pacientes diabéticos.

Asumimos que la respuesta alterada a la hipoxemia debida a la NAC y un comando respiratorio neurológico central no-funcional podrían tener un papel clave en estos casos de muerte súbita, y dicha suposición es la base de este breve reporte sobre eventos cardiorrespiratorios graves y súbitos, sin signos clínicos asociados, en dos pacientes diabéticas tipo 1 con NAC grave hospitalizadas en nuestro departamento.

\section{Presentación de casos}

Se emplearon las pruebas de Ewing para diagnosticar NAC en nuestras dos pacientes. Se midió la respuesta de la frecuencia cardiaca a una respiración profunda y al asumir la posición de pie, mediante un electrocardiograma. La respuesta de la presión arterial al asumir la posición de pie se midió automáticamente con Dinamap ${ }^{\circledR}$. No fue posible aplicar la prueba de Valsalva debido a la presencia de retinopatía proliferativa en las dos pacientes.
La primera paciente era una mujer de 54 años, en terapia con insulina desde el año 2000, cuando se diagnosticó diabetes tipo 1 en el contexto del síndrome de poliuria-polidipsia. Desde entonces, su diabetes estuvo mal controlada, con múltiples hospitalizaciones por cetoacidosis y niveles de HbAlc crónicamente elevados entre 11 y $15 \%$ (97 y $140 \mathrm{mmol} / \mathrm{mol}$, respectivamente). Se desarrollaron complicaciones de la diabetes, incluyendo retinopatía diabética que requirió tratamiento con láser y enfermedad renal crónica en etapa terminal, que requirió diálisis. Sin embargo, la paciente no tenía historial de insuficiencia cardiaca o respiratoria. No fumaba; su evaluación cardiovascular más reciente, de 2015, no reportó evidencia de ateroma en los troncos supraaórticos, y su puntaje de calcio coronario fue bajo, igual a 14 .

La segunda paciente era una mujer de 55 años con diabetes tipo 1. Su diabetes se diagnosticó en 1973 en relación con un coma cetoácido. Se inició la terapia con insulina inmediatamente. Puesto que su diabetes comenzó en la niñez temprana y estuvo mal controlada, la paciente desarrolló asimismo retinopatía grave, que se trató, así como insuficiencia renal de grado terminal, que requirió trasplante renal. La paciente presentaba muchas comorbilidades cardiovasculares, incluyendo cardiomiopatía isquémica con disfunción ventricular izquierda, múltiples intervenciones de stent y bypass en los miembros inferiores, hipertensión y amputación transtibial bilateral. En contraste, hasta el momento no se había detectado deterioro en la condición respiratoria.

Ambas pacientes ingresaron en la unidad de cuidado podiátrico del Departamento de Diabetología en el Hospital Pitié-Salpêtrière (París, Francia) debido a una herida infectada en un miembro inferior, localizada en el talón izquierdo y en el muñón del miembro inferior derecho, respectivamente.

Como en cualquier paciente recién ingresado, se practicó un tamizaje para NAC en ambas pacientes, y se confirmó que era grave, de acuerdo con la clasificación de Ewing. De hecho, ambas mostraron hipotensión ortostática asociada con una respuesta inapropiada en la frecuencia cardiaca al adoptar la posición de pie y a la respiración profunda.

El denominador común en los dos casos clínicos fue una baja saturación sanguínea aislada detectada en el oxímetro de pulso durante la revisión rutinaria ( $77 \%$ y $85 \%$, respectivamente), en contraste con la ausencia de molestias como disnea, polipnea o signos de insuficiencia respiratoria, según se observó en el examen clínico (Tabla 1). Cabe señalar que la gasometría arterial confirmó subsecuentemente la baja saturación de oxígeno en la sangre, y que ambas pacientes se hallaban hipoxémicas, de hecho.

Paciente 1. Desde unos días antes del evento agudo no se realizó una ultrafiltración eficiente debido a la somnolencia de la paciente. La paciente presentó sobrecarga vascular con edema en los miembros inferiores, efusión pleural y episodios graves de hipoxemia, demostrados por una lectura oximétrica de pulso diaria cercana a $90-92 \%$ sin terapia con oxígeno, en contraste con la ausencia de disnea o signos de insuficiencia respiratoria. Se monitoreó de manera cercana la saturación de oxígeno. Minutos después de observarse una saturación de $77 \%$ en el oxímetro de pulso, confirmada por gasometría sanguínea $\left(\mathrm{PaO}_{2}\right.$,
116

Kompass Neumol 2021;3:115-118 DOI: $10.1159 / 000519958$ 
Tabla 1. Características de NAC en pacientes, y resultados clínicos respiratorios durante el episodio

\section{Paciente 1 Paciente 2 Normal}

NAC

FC al respirar profundamente

FC al ponerse de pie

1.05

1.0

\subsection{1}

1.09

$>1.11$

Caída en PA al ponerse de pie

Hipoxemia aguda

$\mathrm{SaO}_{2}(\%)$

$\mathrm{PaO}_{2}(\mathrm{mmHg})$

$\mathrm{PaCO}_{2}(\mathrm{mmHg})$

Frecuencia respiratoria (/min)

Disnea

Dificultad para respirar

Respiración paradójica

Cianosis

NAC, neuropatía autonómica cardiaca; FC: frecuencia cardiaca; PA, presión arterial; $\mathrm{PaO}_{2}$, presión parcial del oxígeno en la sangre arterial; $\mathrm{PaCO}_{2}$, presión parcial del dióxido de carbono en la sangre arterial; $\mathrm{SaO}_{2}$, saturación de oxígeno en el oxímetro de pulso.

$59 \mathrm{mmHg} ; \mathrm{PaCO}_{2}, 55 \mathrm{mmHg}$ ), se encontró a la paciente inconsciente, sin respiración ni pulso cardiaco (ausencia de pulso carotideo o femoral y ausencia de pulso registrado por pletismografía). Al instante se aplicaron compresiones torácicas e intubación endotraqueal para administrar ventilación artificial. Después de dos minutos de resucitación cardiopulmonar, la paciente recuperó espontáneamente el ritmo cardiaco, sin necesidad de choque eléctrico ni de fármacos. Se le transfirió a la unidad de cuidados intensivos. Una TC exploratoria torácica descartó el embolismo pulmonar, pero reportó abundante efusión pleural bilateral. Un ECG sin cambios, un ecocardiograma normal y un resultado negativo de troponina fueron argumentos sólidos contra el infarto al miocardio. La fiebre y los marcadores sanguíneos de inflamación se hallaban ausentes, y tampoco había evidencia de desórdenes metabólicos como dispotasemia o hipoglucemia. Se consideró que el paro cardiaco fue causado probablemente por hipoxemia, en vista de la rápida recuperación observada después de la ventilación, así como los resultados del análisis gasométrico sanguíneo, que indicaron hipoxemia e hipercapnia. Puesto que en los días previos no se habían administrado fármacos con efecto depresor central de la respiración, el principal factor de riesgo remanente para la hipoxemia era la sobrecarga vascular debido a una eliminación insuficiente de volumen por diálisis. La continuación de las sesiones de diálisis permitió reducir progresivamente el volumen (el peso disminuyó de 75.7 a 60.8 $\mathrm{kg}$ ) y corregir la hipoxemia, con una $\mathrm{PaO}_{2} \geq 90$ mmHg según la gasometría sanguínea.

Paciente 2. Un valor bajo de saturación de oxígeno, de $85 \%$, se registró en el oxímetro de pulso durante la revisión rutinaria. No había disnea ni dificultad respiratoria. Se colocó una máscara de oxígeno con un flujo de $9 \mathrm{~L} / \mathrm{min}$, lo que permitió recuperar la saturación a 95\%, según la oximetría de pulso. Se le transfirió a la unidad de cuidados intensivos tras detectar edema pulmonar con efusión pleural mediante examen pulmonar y TC exploratoria torácica. El hallazgo de valores incrementados de troponina, un ECG modificado con depresión del segmento ST e inversión de la onda T en el territorio inferolateral, además de hipocinesia cardiaca en el ecocardiograma, permitió mantener el diagnóstico de edema pulmonar secundario a un síndrome coronario agudo. Puesto que la coronariografía no mostró progresión de las lesiones coronarias, no se realizó procedimiento de revascularización alguno. La paciente recuperó una saturación normal de oxígeno sanguíneo luego de recibir medicación diurética combinada con ventilación no-invasiva y punción pleural evacuatoria. La ecografía mostró una fracción de eyección ventricular de $33 \%$, y se introdujeron $\beta$-bloqueadores en dosis gradualmente crecientes.

\section{Discusión y conclusión}

El denominador común de los dos casos es la ausencia de disnea o de signos precursores del evento cardiorrespiratorio súbito, en contraste con la presencia de hipoxemia confirmada por oximetría de pulso, además de marcadores sanguíneos, y la presencia de una etiología evidente de hipoxemia.

Más aún, en ambos casos, dicha ausencia de signos precursores parecía relacionarse con la gravedad del trastorno autonómico, evidenciado por el tamizaje de NAC. Por esto, el deterioro de la actividad respiratoria hipóxica causado por neuropatía autonómica diabética es altamente sospechoso de causar muerte súbita cardiaca. Nuestras observaciones coinciden con reportes previos sobre muerte súbita en pacientes con NAC. Un metaanálisis reciente de 14 estudios, con un total de 5647 casos de MSC y 346,356 participantes, mostró un riesgo dos veces mayor de MSC en sujetos con diabetes [7]. Se encontró que la presencia de NAC es un factor significativo para tales observaciones. De hecho, en un análisis de 2900 sujetos diabéticos, Vinik et al. demostraron que el subconjunto de pacientes con NAC (definido por un resultado anormal en las pruebas de Ewing) mostró un aumento significativo, de 2.14 veces, en el riesgo relativo de muerte, que incluso subió a 3.65 si el NAC se definía como la presencia de resultados anormales en más de dos pruebas cuantitativas de función autonómica [5], mostrando así una clara relación entre la gravedad de la NAC y la mortalidad. Varios estudios realizados en la década de 1990 pusieron de manifiesto que la disfunción del comando neurológico central de respiración en pacientes con NAC produce hipoxemia. Por ejemplo, se encontró que la apnea del sueño obstructiva central [8] y los episodios de desaturación por debajo de 85\% [9] son más prevalentes en pacientes diabéticos con NAC que en quienes no la padecen. Adicionalmente, numerosos estudios resaltaron también el deterioro en la actividad respiratoria hipóxica en pacientes diabéticos con NAC $[10,11]$. De hecho, en comparación con participantes de control, la sensibilidad barorrefleja basal está debilitada en pacientes diabéticos tipo 1 con NAC. Estos últimos no son capaces de aumentar espontáneamen- 
te su ventilación para combatir la hipoxia, y la administración de oxígeno de corto plazo podía restablecer temporalmente la sensibilidad barorrefleja [12].

Todos esos resultados constituyen un sólido cuerpo de evidencia que sugiere que los pacientes con NAC pierden la capacidad para incrementar su ventilación de manera instantánea debido a la hipoxia. Fisiopatológicamente, los quimiosensores de la actividad respiratoria ya no son estimulados por la hipoxemia. Puesto que quimiosensores como los cuerpos carotideos, sensibles a la presión de oxígeno arterial, son inervados por el sistema parasimpático, los pacientes con NAC grave podrían presentar un deterioro en la actividad respiratoria como respuesta a la hipoxia por una disfunción del sistema nervioso autónomo. Una demora en el manejo de la hipoxemia debido a la ausencia de signos clínicos de alerta podría favorecer la ocurrencia de eventos cardiorrespiratorios súbitos y tener consecuencias dramáticas, como la muerte. El corazón diabético suele acompañarse por un mayor grado de hipoxia en el tejido miocárdico. Como marcador de hipoxia, la expresión de la enzima anhidrasa carbónica se incrementa en la cardiomiopatía isquémica diabética, y media la muerte de células endoteliales y miocitos [13]. Estas observaciones resaltan el papel potencial de la anhidrasa carbónica en los eventos desencadenados por la desregulación que favorece la NAC.

Para concluir, el punto importante es que es fácil pasar por alto la hipoxemia, lo que retrasaría el diagnóstico de enfermedad cardiopulmonar, puesto que en estos dos casos clínicos no se reportaron signos de insuficiencia respiratoria. Deben emprenderse el tamizado sistemático de la NAC en pacientes diabéticos y la detección proactiva del deterioro en la actividad respiratoria hipóxica para manejarla de manera temprana (p.ej., dando tratamiento a la hipoxemia), para prevenir consecuencias dramáticas como el paro cardiorrespiratorio y la muerte. Esto también permitiría un manejo cuidadoso de los medicamentos con efecto depresor de la respiración en esta población con mayor riesgo de MSC, particularmente en casos con enfermedades crónicas cardiacas y pulmonares, bajo anestesia general, con síndrome grave de apnea del sueño o cualquier situación médica que exacerbe la hipoxemia. Las comorbilidades presentes en nuestros dos pacientes representan una limitación de nuestro reporte. No solamente la hipoxemia, sino otras funciones en la fisiología respiratoria podrían asimismo ser factores coadyuvantes para un resultado desfavorable [14], como el paro cardiorrespiratorio.

\section{Disclosure statement}

No hay conflicto de interés en el área de este estudio.

\section{Información sobre licencias}

Louis Schubert, Suzanne Laroche, Agnès Hartemann, Olivier Bourron, Franck Phan: Impaired hypoxic ventilatory drive induced by diabetic autonomic neuropathy, a cause of misdiagnosed severe cardiac events: brief report of two cases. BMC Cardiovasc Disord. 2021 Mar 17;21(1):140 (DOI: 10.1186/s12872-021-01944-4). ${ }^{\circledR} 2021$ Los autores (traducción; abreviaturas, agradecimientos, contribuciones de los autores, financiamiento, disponibilidad de datos y materiales, consentimiento para publicación, autorización ética y consentimiento para participar, nota del editor abreviadas), protegido por CC BY 4.0 (https://creativecommons.org/licenses/by/4.0/ deed.es).

\section{Referencias}

Las referencias están disponibles en www.karger.com/Article/Fulltext/ 000519958 . 